TRIB2004-64334

\title{
VISION-BASED MICROTRIBOLOGICAL CHARACTERIZATION OF LINEAR MICROBALL BEARINGS
}

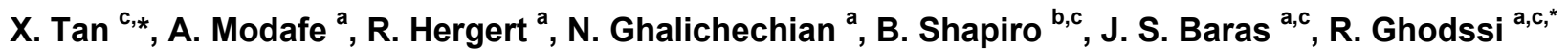 \\ ${ }^{a}$ Department of Electrical and Computer Engineering \\ ${ }^{b}$ Department of Aerospace Engineering \\ ${ }^{\mathrm{C}}$ Institute for Systems Research \\ University of Maryland, College Park, MD, 20742
}

\begin{abstract}
Microball bearings can potentially provide robust and low friction support in micromachines such as micromotors and microgenerators. Their microtribological behavior needs to be investigated for design and control of such micromachines. In this paper a vision-based, non-intrusive measurement method is presented for characterization of friction in linear microball bearings. Infrared imaging is used to directly observe the dynamics of microballs and track the motion of bearing components. It is verified that microballs roll most of the time with occasional sliding or bumping resulting from fabrication nonuniformity. The friction-velocity curve demonstrates evident hysteresis. The dependence of frictional behavior on several factors is studied.
\end{abstract}

\section{INTRODUCTION}

Microball bearings have potential to offer robust and low friction support in micromachines, such as micromotors and microengines. A careful study on their microtribological behavior is necessary for proper design and effective control of micromachines based on such bearings. A linear microball bearing structure was proposed and its static coefficient of friction (COF) measured by Ghodssi et al [1]. A vision-based experimental setup was developed to study the dynamic friction of linear ball bearings by Lin et al [2], where the underlying friction model consisted of the Coulomb friction only.

As a continuation of the work in [2], this paper contains indepth characterization and understanding of the microtribological behavior using infrared imaging, scanning electron microscopy (SEM) imaging, and analysis. The contributions of this paper include: a) verification of rolling motions of microballs by directly observing trajectories of all bearing elements (stator, slider, balls); b) observation of the hysteresis between the friction and the relative velocity; and c) investigation of the influences of the ball number and oxide formation on the frictional behavior.

* Corresponding authors. X. Tan is currently affiliated with the Department of Electrical and Computer Engineering, Michigan State University, East Lansing, MI, 48824. Email: xtan@egr.msu.edu (X.Tan), ghodssi@eng.umd.edu (R. Ghodssi).

\section{EXPERIMENTAL SETUP}

A schematic of linear microball bearings is shown in Fig.1. The bearing consists of two silicon plates (slider and stator) and stainless steel microballs of diameter $285 \mu \mathrm{m}$. Two parallel Vgrooves, which house the balls, are etched on the plates using potassium hydroxide $(\mathrm{KOH})$. In the experiments the stator of the bearing is mounted on an oscillating platform driven by a servo motor. The only force acting on the slider is the friction applied by the microballs. By collecting motion data of the bearing elements through a CCD camera, one can infer the friction dynamics inside the system. For a more detailed description of the basic setup, see [2]. Several improvements have been made to upgrade the system reported in [2]. A camera (Sony DCR-TRV22) with night-shot mode, a finepositioning stage, a $24 \mathrm{X}$ magnification lens, and a halogen bulb as an infrared source enable one to see through the slider and observe the microballs closely. Figure 2 shows a picture of the experimental setup.

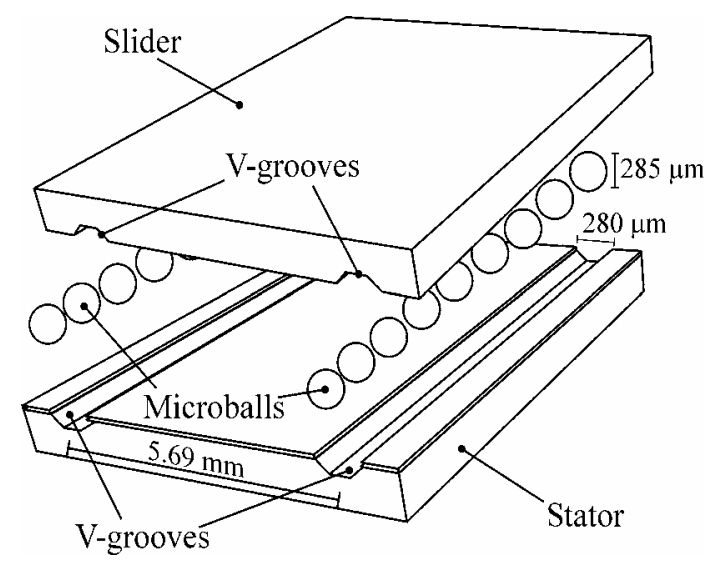

Fig. 1. A schematic diagram of linear microball bearings [2]. 


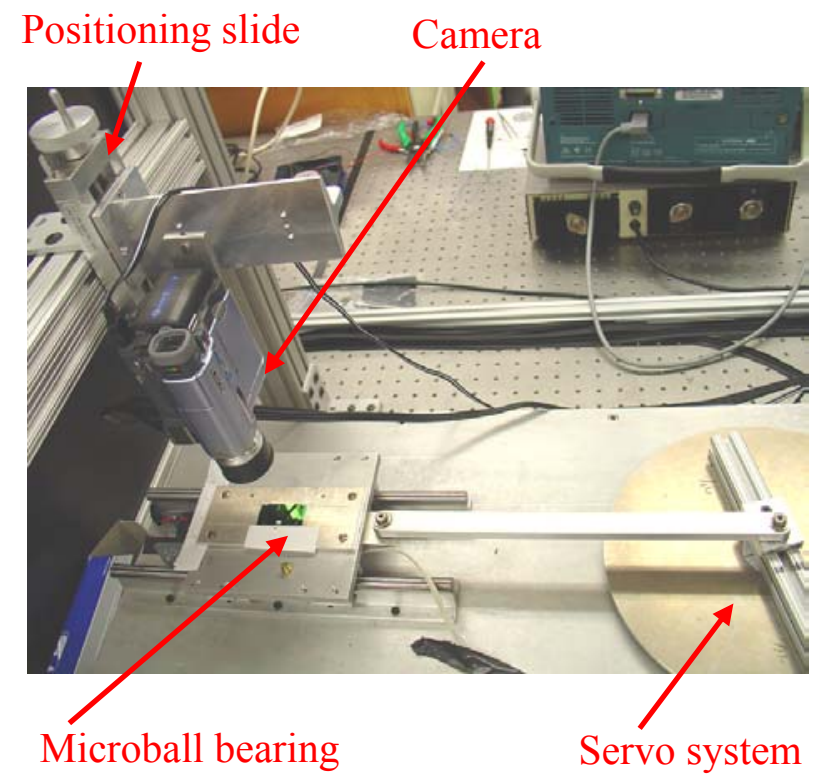

Fig. 2. The experimental setup.

\section{EXPERIMENTAL RESULTS AND DISCUSSIONS Rolling Motion}

Low friction in ball bearings arises from the rolling motion of the balls. Hence it is of interest to study whether the microballs roll (instead of slip) in microball bearings. Consider Fig. 3. The condition for the ball to roll on both surfaces is that the relative velocities at the contact points $\mathrm{A}$ and $\mathrm{B}$ are zero. Let $\omega$ be the angular velocity of the ball and $r$ be its radius. Let $V_{b}$ and $V_{p}$ be the linear velocities of the ball and of the top plate with respect to the bottom plate, respectively. Then the rolling condition implies: $V_{b}=\omega r$, and $V_{p}=V_{b}+\omega r=2 V_{b}$.

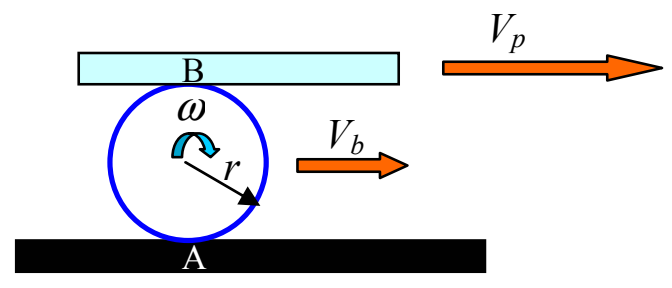

Fig. 3. Illustration of rolling.

In our experiments it is difficult to fully verify the rolling motion of the balls since their angular velocities cannot be derived from the 2D images. However, one can verify the second condition $V_{p}=2 V_{b}$ using the available information, as described below.

For tracking of all the bearing elements different marks were also etched close to the $\mathrm{V}$-grooves on the slider and the stator. Figure 4 shows an infrared image taken under the nightshot mode when the setup is stationary. The different shapes of the stator marks, slider marks, and projection images of balls can be recognized through image processing, which provides the absolute locations of these elements. Figure 5 shows an image where the bearing elements are in motion, and it is blurred due to the relatively low capturing rate of the camera
(30 frames/second), which is the major source of measurement error. In this experiment the exact trajectory of the stator can be pre-determined. By comparing this with the measured trajectory obtained through image processing, we calculated the average position measurement error to be $16 \mu \mathrm{m}$, and the average error in the velocity to be $1 \mathrm{~mm} / \mathrm{s}$ (and hence the error in relative velocity is $2 \mathrm{~mm} / \mathrm{s}$ ).

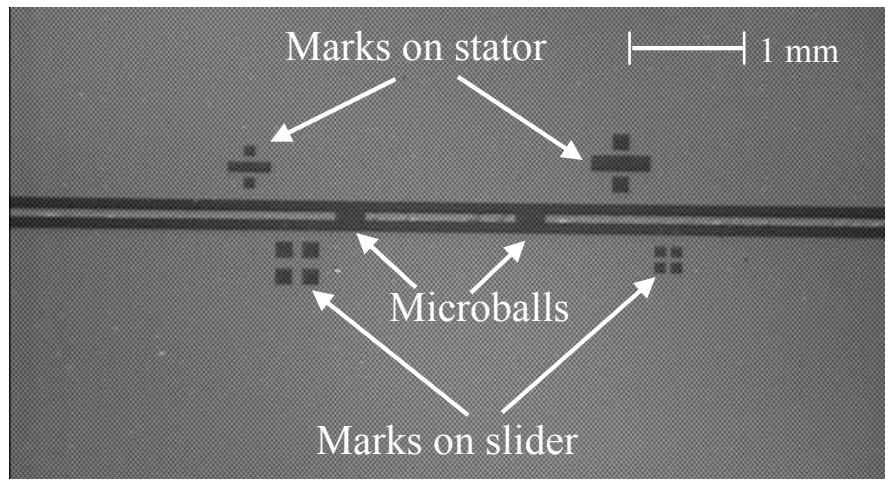

Fig.4. Infrared imaging of stationary bearing elements.

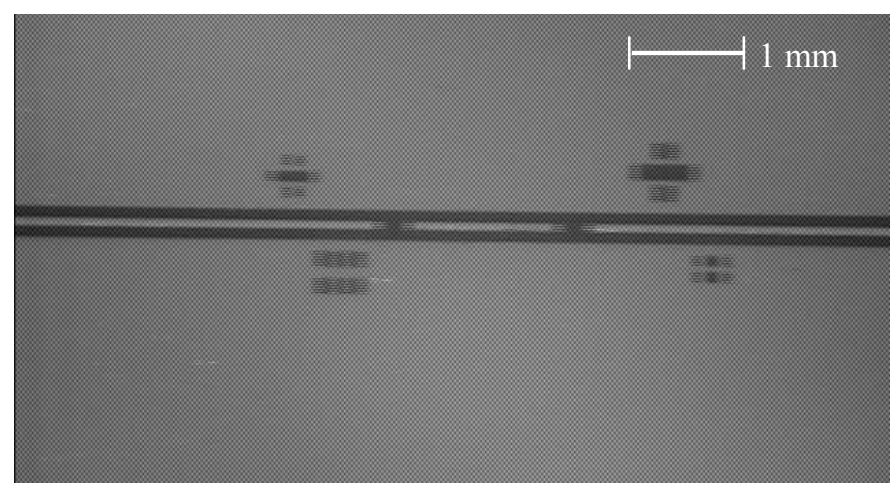

Fig.5 Infrared imaging of moving bearing elements.

Figure 6 shows the trajectories of the stator, the slider, and the two balls (ball 1 and ball 2), where the excitation frequency of the stator is $3 \mathrm{~Hz}$. Figure 7 compares the relative velocity of the slider and twice of those of ball 1 and ball 2 (all with respect to the stator)(top plot and middle plot), and shows their errors (bottom plot). It can be seen that most of the time the errors lie in the range of measurement tolerance, which strongly indicates that the balls roll on both contact surfaces at those times. However, both errors at $\mathrm{t}=1.3 \mathrm{~s}$ exceed the tolerance level, which is an indication of some impact. This is further verified by the sudden change of the slider's acceleration (namely the frictional force normalized by the slider mass), as shown in Fig. 8.

Figure 9 shows the evolution of the measured distance between ball 1 and ball 2. Taking into account the measurement error, it can be seen that the inter-ball distance varies. The reason behind this is the imperfect wall surfaces of the $\mathrm{V}$ grooves. The $\mathrm{KOH}$ etching process leaves level jumps and hillocks on the walls, which prevents balls from uniform, 
synchronized, and constant rolling. This is seen in Fig. 10, an SEM image of the V-groove wall surface. The nonuniformity on the sidewall and surface compliance lead to an uneven contact stripe instead of a contact line between the slider/stator and the balls, as clearly seen in Fig. 11 .

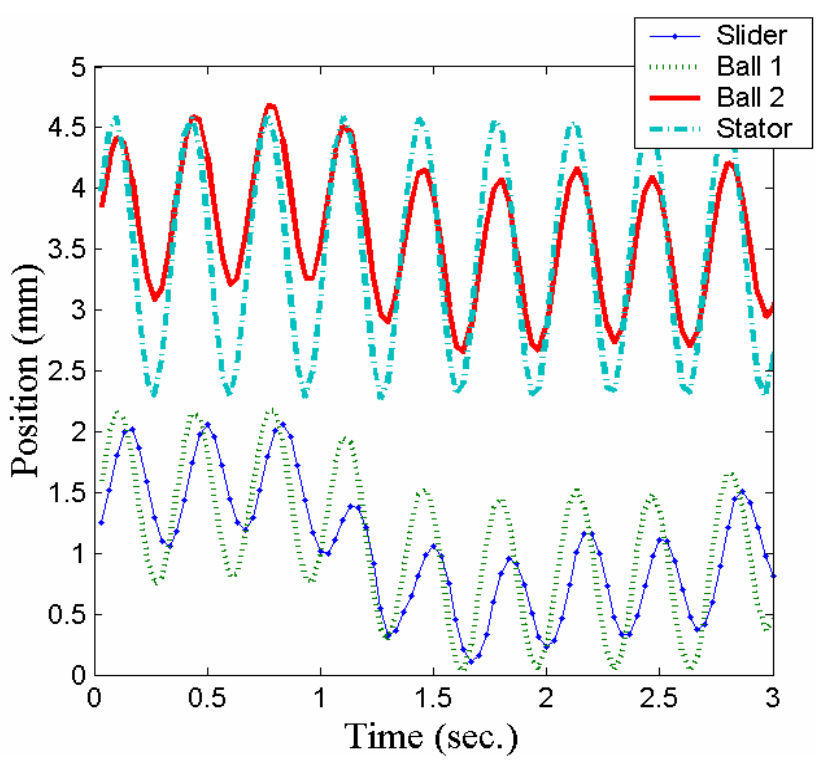

Fig. 6. Measured rajectories of the stator, the slider, and the two balls.
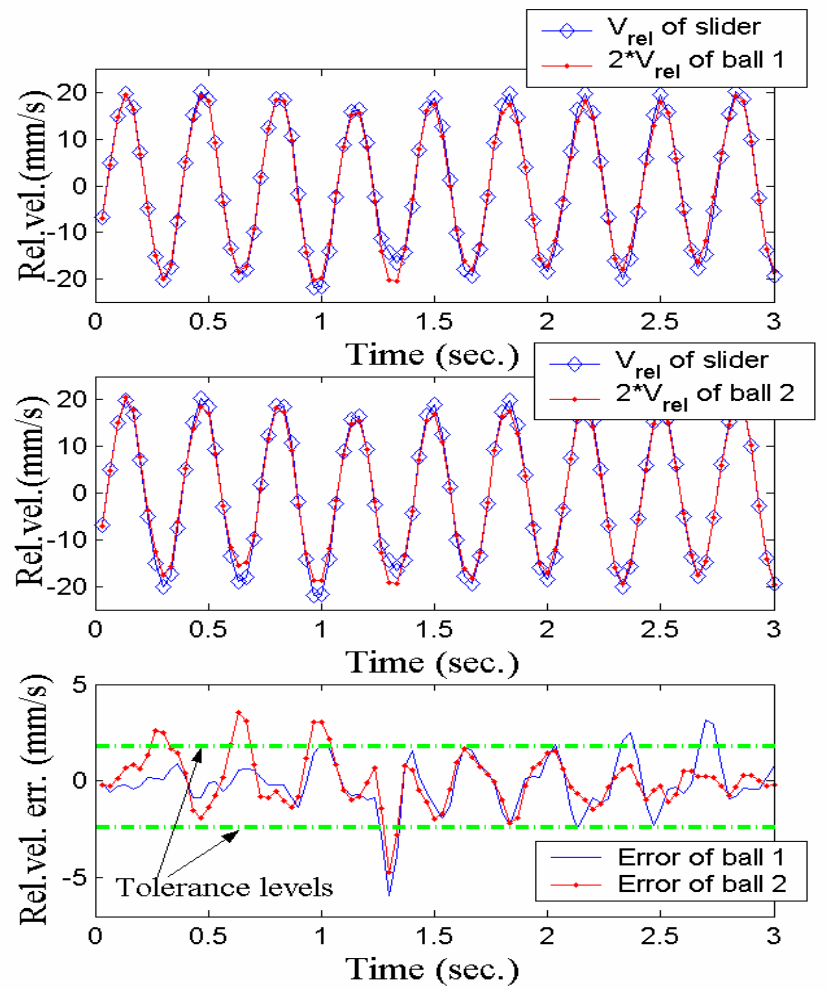

Fig. 7. The relative velocities of the slider and the two balls with respect to the stator.

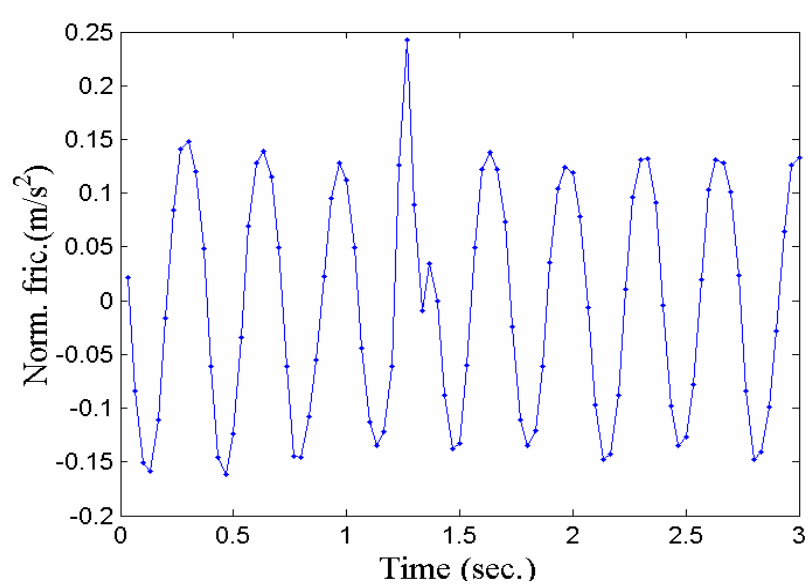

Fig. 8. The normalized friction experienced by the slider (i.e., the acceleration).

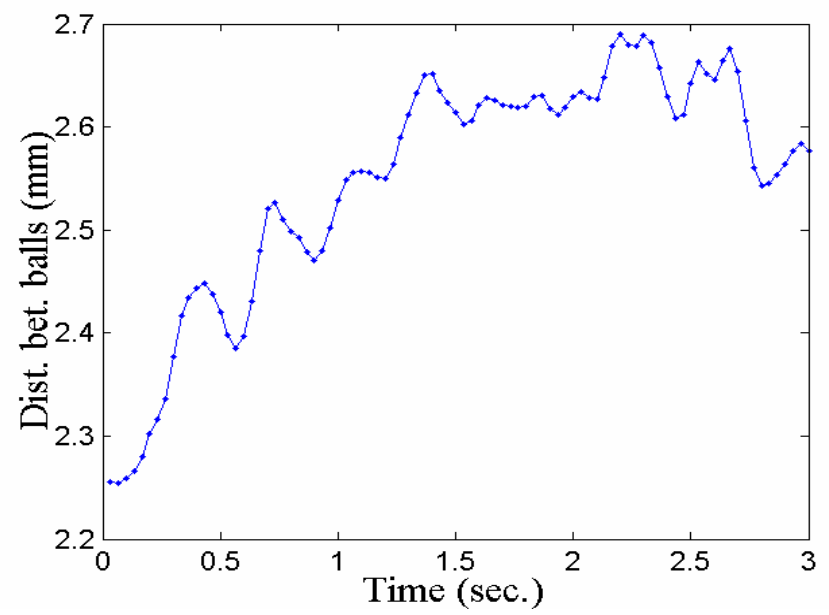

Fig. 9. Evolution of the distance between ball 1 and ball 2 .

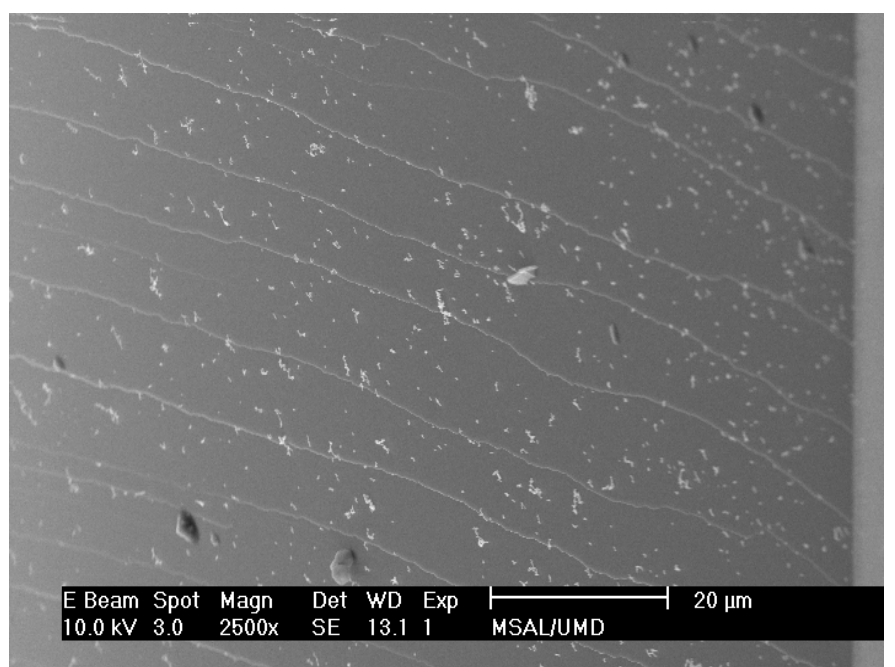

Fig. 10. An SEM image of an unused V-groove sidewall showing level jumps (every about $10 \mu \mathrm{m}$ ) and hillocks (sizes of several $\mu \mathrm{m}$ ). 


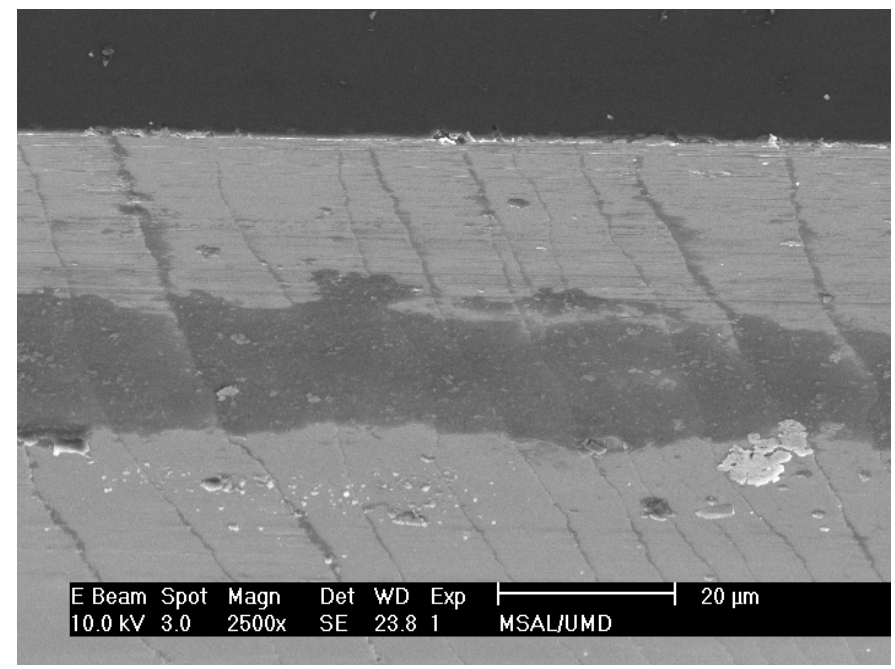

Fig. 11. Contact trace left by microballs on the V-groove sidewall.

\section{Hysteresis}

Classical frictional models typically express the frictional force as a static function of the relative velocity between the contact surfaces, which may include the static friction, Coulomb friction, Stribeck friction, and viscous friction [3-5]. The Dahl model describes the friction in the stiction regime (also called presliding regime or micro-slip regime) in terms of the micro-displacement [6]. The relationship between the friction and the displacement can be hysteretic [7]. A dynamic friction model (called LuGre model) was presented in [8] with an internal state representing the average deflection of contacting asperities. This model demonstrated, among other properties, the hysteresis between the friction and the sliding velocity for unidirectional sliding (i.e., no velocity reversal). It is extended in [7] so that the presliding hysteresis is accounted for explicitly. The friction-velocity hysteresis was also reported in [9] for unidirectional, unsteady sliding velocities and modeled through a time lag. Similar hysteresis behavior was studied for a forced oscillator with a compliant contact [10]. The experimental results reported in [7-10] were all based on macroscopic machines or systems.

The friction-velocity hysteresis is also observed in linear microball bearings. Figure 12 depicts the normalized friction (i.e., slider acceleration) versus the relative velocity of the slider with respect to the stator (3 cycles plotted), where the hysteresis is evident. While an in-depth understanding and modeling of this phenomenon is underway, the strong adhesion force (comparing to the gravity) experienced by the microballs may play a role here. The adhesion force acts as a nonlinear spring at (relative) velocity reversals, and its interaction with the oscillating stator can lead to hysteresis [10]. Figure 13 shows a microball sticking to the V-groove sidewall on the slider. The slider is almost perpendicular to the horizontal plane. This demonstrates the magnitude of adhesion force in comparison with the gravity.

\section{Factors Affecting the Friction}

The effects of the number of balls, and the native oxide formation on friction are investigated.

\section{Number of Balls}

More balls tend to provide better contact between the slider and the stator. However, it makes the $\mathrm{V}$-grooves more crowded and creates more ball to ball or ball to end-wall collisions, thus rendering the contact dynamics more complicated. Figure 14 shows the comparison of normalized friction trajectories for the cases where 3 balls per groove and 6 balls per groove were used, respectively, with an excitation frequency of $2 \mathrm{~Hz}$. It can be seen that with 6 balls per groove the friction level shows much more variation. This brings up two interesting issues in design and control: a) optimal number of balls given the bearing geometry; and b) adaptive identification of friction model parameters.

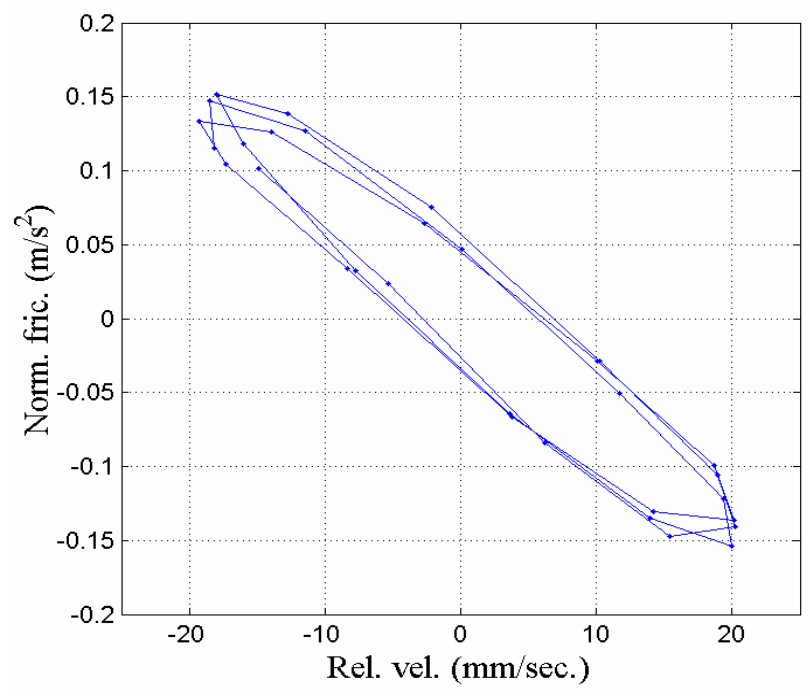

Fig.12. Hysteresis between the friction and the relative velocity (3 cycles), where the excitation frequency is $3 \mathrm{~Hz}$.

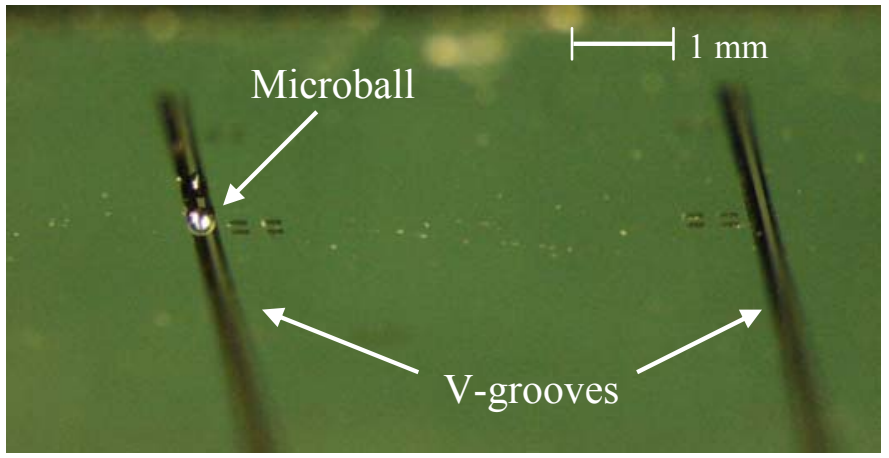

Fig. 13. A microball sticking to the V-groove of the slider, and the slider making an angle of 80 degrees to the horizontal plane. Picture taken under an optical microscope. 

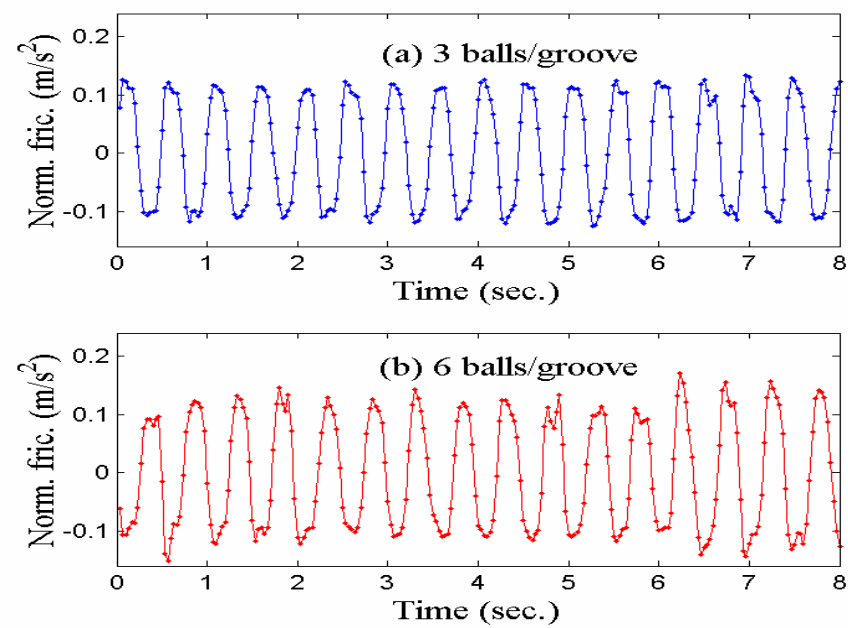

Fig. 14. Friction patterns and levels for a) 3 balls per V-groove; b) 6 balls per groove. Excitation frequency: $2 \mathrm{~Hz}$.

\section{Oxide Formation}

It has been observed that the magnitude of friction varies significantly from time to time. A more careful study reveals that the friction depends strongly on how long the bearing has been exposed to the air. In all the experiments performed, the ambient temperature was about $25^{\circ} \mathrm{C}$ and the relative humidity was about $40 \%$. We first let the bearing sit for hours and then performed an experiment to measure the friction. The slider was then gently rubbed against the stator manually for 5 seconds, and the same friction experiment was conducted. Figure 15 compares the measured friction in the two experiments, and one can see that in the latter the friction was much lower and more regular. The reduction of friction is believed to arise from partial removal of the native oxide on the contact stripes. Figures 16 and 17 show the SEM images of the contact stripe before and after rubbing.
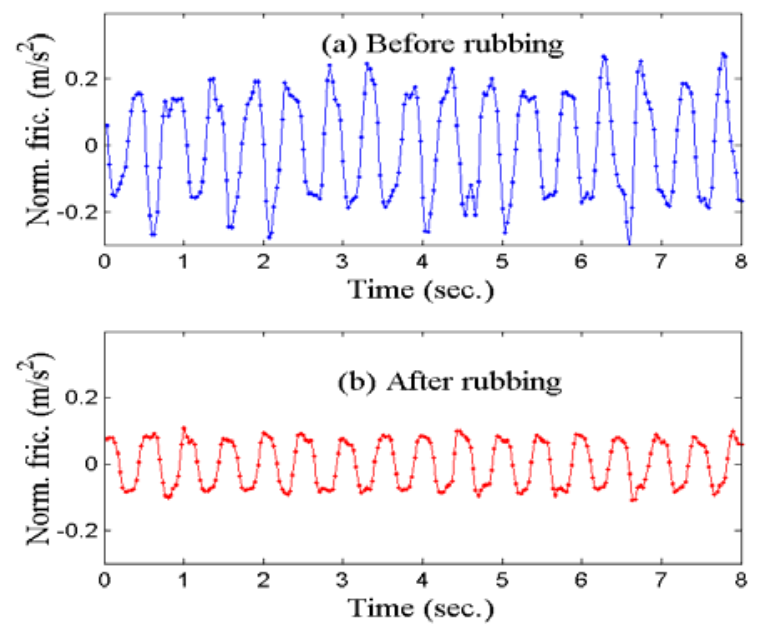

Fig. 15. Comparison of friction before and after manually rubbing the slider against stator.

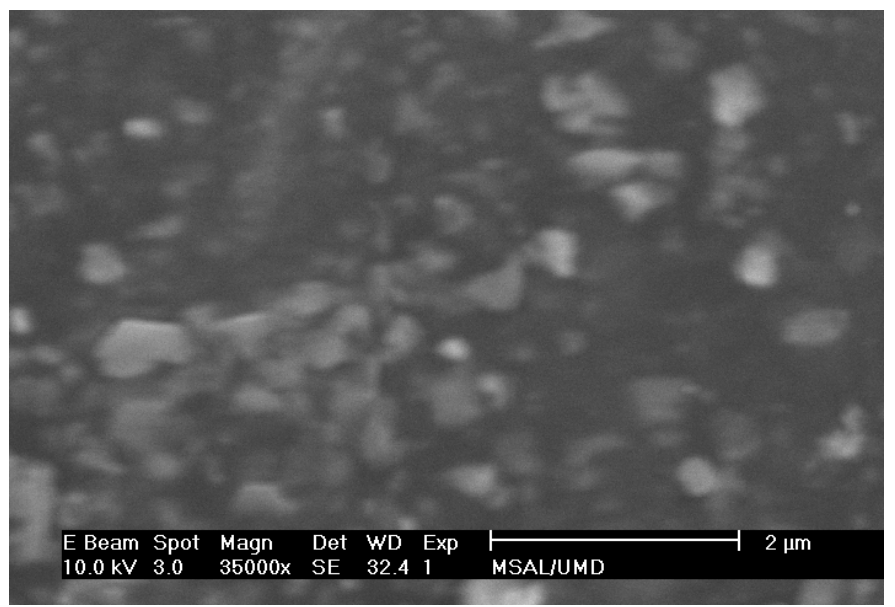

Fig. 16. SEM image of the contact stripe before rubbing.

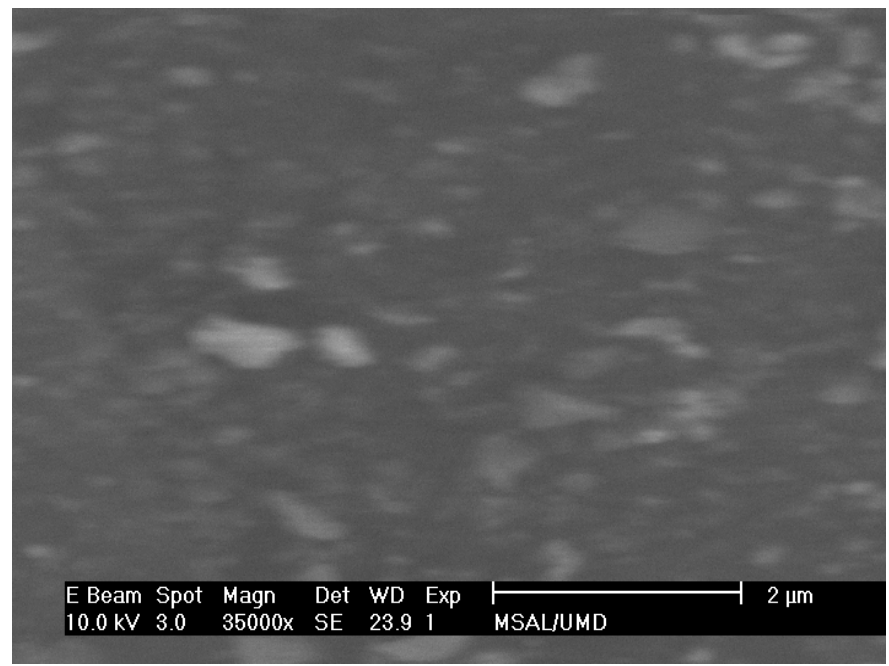

Fig. 17. SEM image of the contact stripe after rubbing.

The experiments suggested that native oxide growth may prevent the bare-silicon bearing from achieving very low and consistent friction. Therefore, an appropriate coating (e.g., diamond or silicon carbide) on the bearing surface would be necessary for long-term, steady performance of these microball bearings.

\section{CONCLUSIONS}

In this paper infrared imaging-based tribological study is reported for a linear microball bearing. Dynamics of the bearing elements were directly observed, measured, and analyzed. General rolling motion was verified leading to low average friction. Occasional micro-slip and impacts on balls were also recorded. Other interesting phenomena, such as the friction-velocity hysteresis, and the influences of several factors on friction were presented. Several design and control issues were raised along with the discussions. For future work, the use of a high speed, infrared camera ( $>500$ frames/second) would 
enhance the measurement precision and thus allow a study at much higher excitation frequencies.

\section{ACKNOWLEDGMENT}

The first author would like to acknowledge useful discussions with Prof. P. S. Krishnaprasad. The authors would like to thank Wen-Hsien Chuang for his assistance in taking the SEM pictures. This work was supported in part by the NSF grant ECS-0224361 and by the Lockheed Martin Chair Endowment Fund.

\section{REFERENCES}

[1] Ghodssi, R., Denton, D. D., Seireg, A. A., and Howland, B., 1993, "Rolling Friction in a Linear Microstructure," Journal of Vacuum Science and Technology A, 11, pp. 803-807.

[2] Lin, T-W., Modafe, A., Shapiro, B., and Ghodssi, R., 2004, "Characterization of Dynamic Friction in MEMS-Based Microball Bearings," IEEE Transactions on Instrumentation and Measurement, 53 (3), pp. 839-846.

[3] Armstrong-Helouvry, B., 1991, Control of Machines with Friction, Kluwer Academic Press, Boston, MA

[4] Leonard N., and Krishnaprasad, P. S., 1992, "Adaptive Friction Compensation for Bidirectional Low Velocity
Tracking," Proceedings of the $31^{\text {st }}$ IEEE Conference on Decision and Control, Tucson, AZ, pp. 267-273.

[5] Armstrong-Helouvry, B., Dupont, P., and Canudas de Wit, C., 1994, "A Survey of Models, Analysis Tools and Compensation Methods for the Control of Machines with Friction," Automatica, 30 (7), pp. 1083-1138.

[6] Dahl, P. R., 1968, “A Solid Friction Model," Technical Report TOR-158(3107-18), The Aerospace Corporation, El Segundo, CA.

[7] Swevers, J., Al-Bender, F., Ganseman, C. G., and Prajogo, T., 2000, "An Integrated Friction Model Structure With Improved Presliding Behavior for Accurate Friction Compensation," IEEE Transactions on Automatic Controk, 45 (4),pp. 675-686.

[8] Canudas de Wit, C., Olsson, H., Astrom, K. J., and Lischinsky, P., 1995, "A New Model for Control of Systems With Friction," IEEE Transactions on Automatic Control, 40 (3), pp. 419-425.

[9] Hess, D.P., and Soom, A., 1990, "Friction at a Lubricated Line Contact Operating at Oscillating Sliding Velocities," Journal of Tribology, 112, pp. 147-152.

[10] Liang., J.-W., and Feeny, B. F., 1998, "Dynamical Friction Behavior in a Forced Oscillator With a Compliant Contact," Journal of Applied Mechanics, Transactions of the ASME, 65, pp. 250-257. 